\title{
EDITORIAL
}

\section{Being more positive about negative ventilation?}

\author{
M. Wysocki
}

Mechanical ventilation is undoubtedly a lifesaving procedure for patients with acute respiratory failure [1]. Unfortunately the procedure is also associated with a number of unwanted side-effects. Firstly, until recently, mechanical ventilation required endotracheal intubation either through a tracheostomy or with a translaryngeal tube, procedures which can carry some risks $[2,3]$. Secondly, by disrupting the physiological upper airway barrier, endotracheal intubation increases the risk of acquiring bronchopneumonia [4]. Thirdly, discontinuing mechanical ventilation in intubated patients is difficult and may unneccessarily increase the duration of mechanical ventilation [5]. Finally, positive pressure is used to unload the respiratory system resulting in excessive elevation of intrathoracic pressures which can jeopardize the patient's haemodynamic status because of interactions between the heart and the lung. Such high positive intrathoracic pressures may also induce barotrauma and act to worsen pre-existing pulmonary lesions [6]. Since positive-pressure ventilation is far from physiological, a great deal of effort has been expended to reduce the consequent sideeffects [7-9].

Delivering positive-pressure ventilation through a noninvasive interface (noninvasive positive-pressure ventilation (NIPPV)) avoids intubation-related complications, reduces the length of stay in intensive care, and lowers the mortality rate in patients with acute exacerbations of chronic obstructive pulmonary disease (COPD) [10]. NIPPV is also able to reduce the time required to withdraw the patient from the ventilator and has recently been proposed as a powerful method of weaning in COPD patients intubated for an acute exacerbation of their illness [5].

A further advance in reduction of mechanical ventilation-related side-effects might be to avoid supraphysiological positive intrathoracic pressures by using perithoracic negative pressure as proposed by CORRADO et al. [11] in this issue.

Noninvasive negative ventilation (NINV) increases transpulmonary pressure and assists ventilation by applying a negative pressure around the thorax and the abdomen. This is made possible by ventilators which intermittently generate a negative pressure in a space enclosing the thorax and the abdomen. An extensive description of the method and of devices used to provide NINV can be found in a recently published review [12]. Tank ventilators consist of either a tank or cylinder, e.g. the iron lung, which envelope the body up to the neck. NINV using the iron lung was the first body ventilator to receive widespread use and was the main device employed for both acute and long-

Réanimation polyvalente, Institut Mutualiste Montsouris, $42 \mathrm{Bd}$ Jourdan, 75674, Paris, France. Fax: 33140782476. term ventilatory support from 1931 until the late 1950 s [12]. Compared to other devices for NINV such as chest shells or body wraps, the iron lung is probably the most effective.

Using a case-control design, CORRADO et al. [11] compared 26 COPD patients with acute exacerbations treated with NINV using an iron lung (in future, termed cases) with a matching group of 26 patients intubated endotracheally and ventilated with positive-pressure ventilation (henceforth termed controls). In both groups, the patients were hypercapnic and acidotic, and based on a well-designed matching process, cases and controls were not very different (admission values of blood $\mathrm{pH}$ and arterial carbon dioxide tension $\left(\mathrm{Pa}_{\mathrm{a}} \mathrm{CO}_{2}\right)$, the causes triggering the acute respiratory failure, and the Acute Physiology and Chronic Health Evaluation (APACHE) II severity score were not different in the two groups). The main outcomes were the number of treatment failures (defined as the need for endotracheal intubation or death in the cases and as death in the controls) and death. The outcomes were similar in both groups suggesting that NINV was comparable in effectiveness to the more invasive positive ventilation. However, interpretation of the results is hampered by the fact that the study was done retrospectively and the authors used a case-control design which does not permit definitive conclusions to be drawn. Moreover, controls and cases were not treated at the same site or by the same physicians. Controls were treated in an intensive care unit (ICU), while the cases were treated in a respiratory intermediate ICU (RIICU). This is a possible confounding variable since the mode of treatment is not independent of the site of treatment and one is not sure whether patients treated with the iron lung had an outcome comparable to those treated conventionally or whether patients treated in the ICU had comparable outcomes to those treated in the RIICU. In addition, criteria for endotracheal intubation were well defined in patients treated with the iron lung, but not in those treated conventionally. Since endotracheal intubation was an inclusion criteria in patients treated conventionally a selection bias cannot be excluded.

Recent reviews on noninvasive ventilation [1, 13-15] have not been positive about NINV. All considered it to be poorly tolerated, difficult to adjust for individual patients, and time consuming. Unfortunately these points were not clearly addressed by the present study, yet they can be important factors for limiting the use of NINV. However, the study of CORRADO et al. [11] makes it clear that, for avoiding endotracheal intubation in COPD patients with acute exacerbations, negative ventilation may be an alternative to noninvasive positive ventilation. The authors, observed a success rate of $70 \%$ with NINV (close to the success rate observed with NIPPV [15]) suggesting a 
place for negative ventilation "somewhere" in the therapeutic armamentarium used to avoid endotracheal intubation in such patients. Indeed, "somewhere" needs to be further defined, as do the respective places of NINV and NIPPV in treatment. In patients with acute exacerbations of COPD, three randomized studies [10, 16, 17] have demonstrated that the use of NIPPV avoided endotracheal intubation; moreover, two of these studies demonstrated a reduction in mortality with NIPPV [10, 16] and one [10] a reduction in the length of stay in the ICU. They also strongly suggest that in these patients, NIPPV may be better than the conventional approach, including endotracheal intubation. In the study of CORRADO et al. [11], NINV had an efficacy comparable to the conventional approach in COPD patients with acute respiratory failure. Whilst as direct comparison between NIPPV and NINV is lacking and would be helpful to clarify the respective places of NINV and NIPPV, the present study suggests in these patients a strategy where NIPPV is included as a first line ventilatory support, NINV as a rescue and finally endotracheal intubation as the method of last resort.

From a haemodynamic point of view, NINV may be less detrimental [18] and may be better tolerated in patients with a haemodynamic instability sometimes observed in COPD patients with acute exacerbations. Haemodynamic instability is often an exclusion criteria for noninvasive positive ventilation. A recent study failed to demonstrate a beneficial effect of NIPPV in patients with cardiogenic pulmonary oedema and, more importantly, a negative effect was suspected [19]. In such patients, since it generates more physiological airway pressures, NINV may be a haemodynamically well-tolerated alternative to NIPPV as a recent study [18] demonstrated that, by using continuous negative-pressure instead of continuous positive-pressure, oxygen delivery can be improved mainly because of an increase in cardiac index.

Finally, positive-pressure ventilation leads to barotrauma and complex protective measures must be instituted to prevent this $[7,8]$. In the case of NINV, barotrauma is not an issue. Whether it could be used to reduce to prevent ventilator-induced lung injury or to treat patients already suffering from barotrauma is speculative, but should be kept in mind.

To date, negative ventilation has been used mainly in patients with chronic respiratory disorders and various degrees of exacerbation thereof, but other indications should probably be investigated. Clearly, the size and expense of tank ventilators limit more extensive investigation of uses for noninvasive negative ventilation. It also suffers from being an old method of ventilation, physicians being more enthusiastic about more innovative techniques. However, the working principle of noninvasive negative ventilation is still interesting and efforts should be made to provide it more conveniently at the bedside. It will then probably be possible to investigate more extensively the physiological effects of noninvasive negative ventilation and finally to undertake prospective comparison with other ventilatory approaches, particularly with noninvasive positive-pressure ventilation, in patients with acute respiratory failure. Based on such comparative studies it will be possible to clarify the role of noninvasive negative ventilation in the treatment of patients with acute respiratory failure.

\section{References}

1. Tobin MJ. Mechanical ventilation. N Engl J Med 1994; 330: 1056-1061.

2. Schwartz DE, Matthay MA, Cohen NH. Death and other complications of emergency airway management in critically ill adults. A prospective investigation of 297 tracheal intubation. Anesthesiology 1995; 82: 367-376.

3. Stauffer JL, Olson DE, Petty TL. Complications and consequences of endotracheal intubation and tracheotomy. A prospective study of 150 critically ill adult patients. Am J Med 1981; 70: 65-76.

4. Johanson WG, Pierce AK, Sanford JP. Changing pharyngeal bacterial flora of hospitalized patients: emergence of Gram-negative bacilli. N Engl J Med 1969; 281: $1137-$ 1140.

5. Ely EW, Baker AM, Dunagan DP, et al. Effect on the duration of mechanical ventilation of identifying patients capable of breathing spontaneously. N Engl J Med 1996; 335: 1864-1869.

6. Dreyfuss D, Saumon G. Ventilator-induced lung injury: lessons from experimental studies. Am J Respir Crit Care Med 1998; 157: 294-323.

7. Stewart TE, Meade MO, Cook DJ, et al. Evaluation of a ventilation strategy to prevent barotrauma in patients at high risk for acute respiratory distress syndrome. Pressure- and Volume-Limited Ventilation Strategy Group. $N$ Engl J Med 1998; 338: 355-361.

8. Amato MB, Barbas CS, Medeiros DM, et al. Effect of a protective-ventilation strategy on mortality in the acute respiratory distress syndrome. $N$ Engl J Med 1998; 338: 347-354.

9. Weg JG, Anzueto A, Balk RA, et al. The relation of pneumothorax and other air leaks to mortality in the acute respiratory distress syndrome. $N$ Engl J Med 1998; 338: 341-346.

10. Brochard L, Mancebo J, Wysocki M, et al. Noninvasive ventilation for acute exacerbations of chronic obstructive pulmonary disease. N Engl J Med 1995; 333: 817-822.

11. Corrado A, Gorini M, Pelagatti C, et al. Negative pressure ventilation versus conventional mechanical ventilation in the treatment of acute respiratory failure in COPD patients. Eur Respir J 1998; 12: 519-525.

12. Bach JR. Update and perspectives on noninvasive respiratory muscle aids. Part 1: the inspiratory aids. Chest 1994; 105: 1230-1240.

13. Elliott MW, Moxham J. Noninvasive mechanical ventilation by nasal or facial mask. In: Tobin MJ, ed. Principles and Practice of Mechanical Ventilation. New York, McGraw Hill, 1994; pp. 427-454.

14. Hill NS. Noninvasive ventilation. Does it work, for whom, and how? Am Rev Respir Dis 1993; 147: 1050-1055.

15. Hillberg RE, Johnson DC. Noninvasive ventilation. $N$ Engl J Med 1997; 337: 1746-1752.

16. Bott J, Carroll MP, Conway JH, et al. Randomised controlled trial of nasal ventilation in acute ventilatory failure due to chronic obstructive airways disease. Lancet 1993; 341: 1555-1557.

17. Kramer N, Meyer TJ, Meharg J, Cece RD, Hill NS. Randomized, prospective trial of noninvasive positive pressure ventilation in acute respiratory failure. Am J Respir Crit Care Med 1995; 151: 1799-1806.

18. Scholz SE, Knothe C, Thiel A, Hempelmann G. Improved oxygen delivery by positive pressure ventilation with continuous negative external chest pressure. Lancet 1997; 349: 1295-1296.

19. Mehta S, Jay GD, Woolard RH, et al. Randomized, prospective trial of bilevel versus continuous positive airway pressure in acute pulmonary edema. Crit Care Med 1997; 25: 620-628. 